\title{
COMÉRCIO ELETRÔNICO: O VAREJO VIRTUAL BRASILEIRO
}

\author{
Simone Cardoso* \\ Márcia Hiroko Kawamoto** \\ Ely Mitie Massuda***
}

RESUMO: O comércio eletrônico tem se expandido no mundo nos mais diversos segmentos e o Brasil segue essa mesma tendência. Apesar do crescimento mundial do comércio eletrônico, poucos estudos são realizados, em especial sobre o Brasil. Como objetivo descrever evolução e características do varejo virtual brasileiro, a pesquisa foi realizada por meio de acesso aos periódicos científicos nas bases de dados do portal de periódicos da Capes, Emerald, EBSCO, Scopus, Scielo e Proquest, sem delimitação de data de publicação e em português. Também foram utilizados os relatórios que foram popularizados como Webshoppers. Verificou-se que as empresas de comércio eletrônico se concentram na região Sudeste e as categorias mais vendidas são dos setores de saúde, cosméticos e perfumaria além de moda e acessórios. Houve aumento nas vendas do varejo online mesmo em período de crise e instabilidade econômica.

PALAVRAS-CHAVE: Vendas online; E-commerce; Business to Consumer.

\section{ELETRONIC COMMERCE: BRAZILIAN VIRTUAL RETAIL}

\begin{abstract}
ABSTRATCT: Electronic commerce has expanded worldwide in various segments and Brazil follows the same trend. Despite the worldwide growth of electronic commerce, few studies are done, especially on Brazil. In order to describe the evolution and characteristics of Brazilian virtual retail, the research was carried out through access to the scientific journals in the Capes, Emerald, EBSCO, Scopus, Scielo and Proquest journals portal databases, without publication date delimitation, and in Portuguese. We also used the reports that were popularized as Webshoppers. It has been found that e-commerce companies are concentrated in the Southeast

\footnotetext{
"Centro Universitário de Maringá - UniCesumar, Brasil. E-mail: admsimonecardoso@gmail.com

** Secretaria de Estado da Educação do Paraná - Núcleo Reginal de Educação Maringá - PR, Brasil.

*** Docente do Programa de Pós-graduação Stricto sensu em Gestão do Conhecimento nas Organizações (PPGCO) e no Programa de Pós-graduação Stricto sensu em Promoção da Saúde (PPGPS) no Centro Universitário de Maringá - UniCesumar. Pesquisadora do Instituto Cesumar de Ciência, Tecnologia e Inovação (ICETI), Brasil.
} 
region and the best-selling categories are from the health, cosmetics and perfumery sectors besides fashion and accessories. There was an increase in online retail sales even in times of crisis and economic instability.

KEY WORDS: Online sales; E-commerce; Business to Consumer.

\section{INTRODUÇÃO}

O desenvolvimento das tecnologias de informação e comunicação demarcaram a sociedade do conhecimento e provocaram profundas mudanças no mundo de modo que modificaram as formas de relacionamento entre pessoas físicas ou jurídicas. Segundo Kotler, Kartajaya e Setiawan (2010), a nova onda de tecnologia facilita a disseminação de informações, ideias e opinião pública, e permite aos consumidores colaborarem para a criação de valor. Dentro desta perspectiva, o comércio dispõe das tecnologias da informação e está conectado aos seus fornecedores e clientes por intermédio da internet.

A convergência desses elementos viabilizou as trocas comerciais através do comércio eletrônico (CE) que, a princípio, veio a surgir como mais uma alternativa dentro do varejo tradicional, mas que nos últimos anos tem se sobressaído ao apresentar elevado número de faturamento (ALBERTIN, 2016).

$\mathrm{O}$ CE brasileiro tem registrado crescimento e as projeções apontam para a continuidade dessa alta (ALBERTIN, 2016). O avanço tem ocorrido, mesmo diante do cenário de recessão que o Brasil vivencia desde meados de 2014. A economia tem apresentado retração nos mais variados setores, porém, o segmento de varejo online exibe índices positivos (EBIT, 2018).

A utilização do CE tem mudado o comportamento das pessoas ao produzir um novo perfil de consumidores (COELHO; OLIVEIRA; ALMÉRI, 2013), definindo clientes participativos, uma vez que as pessoas estão criando e consumindo notícias, ideias e entretenimento que buscam não apenas por uma satisfação de nível funcional e emocional, mas também espiritual, seja nos produtos ou serviços que escolhem (KOTLER; KARTAJAYA; SETIAWAN, 2010).

O CE já foi visto pelos consumidores com certa desconfiança, mas a ampla 
disponibilidade de informações disponível na internet, assim como as ferramentas de avaliação de serviços e entrega de produtos auxiliaram a construção de um segmento o qual tem se consolidado ao longo dos últimos vinte anos pelo mundo. Por meio dessas ferramentas é possível ao cliente avaliar sua experiência de compra, podendo realizar uma análise das organizações por intermédio de sua própria vivência. Com essa forma de operacionalizar suas tarefas, assim como do próprio cliente produzir informações e se comunicar, somadas a proteção de dados pessoais no ciberespaço, as vendas online têm conquistado credibilidade ao longo dos últimos 15 anos no Brasil (ALBERTIN, 2016).

Apesar do crescimento do CE no mundo e por se tratar de um fenômeno relativamente recente, ainda se tem um reduzido número de publicações científicas, em especial no que se refere ao tema no Brasil. Portanto, o objetivo deste estudo consistiu em descrever evolução e características do varejo virtual brasileiro.

\section{MATERIAL E MÉTODOS}

Foram realizadas pesquisas off-line e por meios eletrônicos entre o período de janeiro e fevereiro de 2017, por meio de acesso aos periódicos científicos nas bases de dados do portal de periódicos da Capes, Emerald, EBSCO, Scopus, Scielo e Proquest, sem delimitação de data de publicação e em português. Também foram utilizados os relatórios que foram popularizados como Webshoppers e disponibilizados de forma gratuita.

No início dos anos 2000, a empresa Ebit passou a levantar dados e criar relatórios sobre o comércio eletrônico brasileiro, atuando e acompanhando a evolução do varejo virtual brasileiro, tornando-se referência no CE do Brasil. Os relatórios são denominados por Webshoppers cujos objetivos são de "difundir informações para o entendimento do comportamento do indivíduo com o e-commerce e procurar encontrar pontos a serem melhorados no desenvolvimento do comércio eletrônico nacional" (LAS CASAS; PINHEIRO, 2013, p. 48). Os dados são colhidos através de questionários enviados aos clientes virtuais (NASCIMENTO, 2011).

Os dados captados anualmente pela Ebit são divulgados em dois relatórios, 
um referente ao primeiro semestre e outro com dados do segundo semestre de cada ano pesquisado. Esses relatórios são divulgados sobre o primeiro e segundo semestre devido a tendência de comportamento de compra do consumidor ser distinta entre esses dois períodos devido a sazonalidade de consumo. Dessa forma, a presente pesquisa foi realizada com dados relativos ao primeiro semestre devido a disponibilidade de dados referente ao primeiro semestre dos anos de 2001 ao primeiro semestre de 2018.

Os dados compilados foram analisados por meio de frequência absoluta e relativa com base nos dados disponibilizados e coletados dos relatórios Webshoppers. Dados referentes a valores monetários foram atualizados para agosto de 2018 através da página da internet da Fundação de Economia e Estatística Siegfried Emanuel Heuser (FEE, 2017) que utiliza o Índice de Preços ao Consumidor Amplo (IPCA) e o Índice Nacional de Preços ao Consumidor (INPC) do Instituto Brasileiro de Geografia e Estatística (IBGE), o Índice Geral de Preços - Disponiblidade Interna (IGP-DI) e Índice Nacional de Preços da Construção Ciivil (INCC) da Fundação Getúlio Vargas como indexadores. O programa Excel foi utilizado para a confeção dos gráficos e tabelas.

\section{RESULTADOS E DISCUSSÃO}

\subsection{O COMÉRCIO ELETRÔNICO}

A acessibilidade das informações tem alcançado um maior número de pessoas pelo mundo e, mesmo entre populações mais pobres do mundo, por volta de $70 \%$ das pessoas possuem acesso à telefonia móvel, destacando-se que "oito em cada dez habitantes do mundo em desenvolvimento têm telefone celular, e esse número está crescendo continuamente" (WORLD BANK, 2016, p. 6).

$\mathrm{O} C E$, que também é descrito como varejo virtual ou por seu termo na língua inglesa e-commerce, desde então cresceu, profissionalizou-se e tem se tornado uma ferramenta estratégica para as organizações, sejam elas de alcance mundial, como a AliExpress, uma das maiores lojas online existente no mundo ou de pequeno porte 
atendendo a mercados de nicho.

A respeito da definição de CE, Albertin (2010, p. 03) afirma que:

O comércio eletrônico é a realização de toda a cadeia de valor dos processos de negócio num ambiente eletrônico, por meio da aplicação intensa das tecnologias de comunicação e de informação, atendendo aos objetivos de negócio. Os processos podem ser realizados de forma completa ou parcial, incluindo as transações negócio-a-negócio, negócio-a-consumidor e intraorganizacional, numa infraestrutura predominantemente pública de fácil e livre acesso e baixo custo.

As principais definições de CE estão fundamentadas na comercialização e venda de produtos ou serviços, envolvendo a cadeia de valor dos processos de negócio, em um ambiente eletrônico. A popularização do uso das tecnologias de comunicação e informação tem contribuído para o desenvolvimento das atividades que envolvem o CE (COELHO; OLIVEIRA; ALMÉRI, 2013).

Existem definições para o CE as quais foram se complementando ao longo dos anos, conforme o Quadro 1:

Quadro 1. Definições para comércio eletrônico

\begin{tabular}{|l|l|}
\hline Autor & \multicolumn{1}{c|}{ Conceito } \\
\hline $\begin{array}{l}\text { Kalakota e Whinston } \\
(1997)\end{array}$ & $\begin{array}{l}\text { Uma forma em que as empresas buscam incrementar a eficiência das } \\
\text { comunicaçóes de negócio para expandir a participação no mercado e } \\
\text { manter a viabilidade de longo prazo no ambiente de negócio que existe } \\
\text { hoje. }\end{array}$ \\
\hline \multirow{2}{*}{ Venetianer (2000) } & $\begin{array}{l}\text { Conjunto de todas as transações comerciais efetuadas por uma empresa, } \\
\text { visando atender, direta ou indiretamente, a um grupo de clientes, } \\
\text { utilizando, para tanto, as facilidades de comunicação e de transferência } \\
\text { de dados mediados pela rede mundial de computadores, a internet. }\end{array}$ \\
\hline
\end{tabular}




\begin{tabular}{|l|l|}
\hline Bertaglia (2009) & $\begin{array}{l}\text { Meio pelo qual as empresas podem se relacionar comercialmente com } \\
\text { seus fornecedores, clientes e consumidores em escalas maiores do que as } \\
\text { tradicionais. Inclui pesquisa, desenvolvimento, marketing, propaganda, } \\
\text { negociação, vendas e suporte. }\end{array}$ \\
\hline Kotler (2006) & $\begin{array}{l}\text { Ampla variedade de transaçôes eletrônicas, tais como o envio de pedidos } \\
\text { de compra para fornecedores via EDI (troca eletrônica de dados). Por } \\
\text { meio do comércio eletrônico, os clientes podem projetar, solicitar } \\
\text { produtos e serviços e pagar por eles por vias eletrônicas; e com os } \\
\text { serviços de entrega receberem seus produtos no local cadastrado para } \\
\text { recebimento. }\end{array}$ \\
\hline
\end{tabular}

Fonte: Elaborado pelos autores.

No CE existem diferente formas de atuação das lojas virtuais, podendo ser de e-commerce puro, onde se realizam operações comerciais somente por meio de vendas online. O outro tipo é o misto, em que além das vendas por meio de plataforma de e-commerce, existe também ponto físico de venda. $\mathrm{Na}$ atualidade, lojas de e-commerce puro demonstram tendência de crescimento frente as lojas de e-commerce misto (SEBRAE, 2016; EBIT, 2017).

Existem quatro principais tipos de comércio ou de negócios (CATALANI et al., 2008; SCANDIUZI; OLIVEIRA; ARAÚJO, 2011). Observam-se no Quadro 2, as principais características de cada um desses tipos de CE. 
Quadro 2. Tipos de comércio eletrônico



Fonte: Adaptado de Catalani et al. (2008), Scandiuzzi, Oliveira e Araújo (2011).

Existem tipos que não possuem um alcance maior de popularização entre os usuários: o business-to-empoloyee - $\mathrm{B} 2 \mathrm{E}$, que "enfatizam iniciativas de empresas voltadas para os empregados"; o G2B/G2C que envolve transações entre empresas privadas e o governo, ou entre o governo e consumidor final, que são "interações eletrônicas entre o governo e as empresas (vice-versa) e também entre consumidores finais" (CATALANI et al., 2008, p. 39). No presente artigo, foi abordado o tipo de comércio eletrônico B2C. 


\subsection{A EVOLUÇÃO DO COMÉRCIO ELETRÔNICO}

Foi em 1982 que aconteceu a primeira transação via web, quando a agência de viagens inglesa conseguiu extrair dados virtualmente e responder prontamente ao usuário (ABE, 2018). Na década de 1990, o CE expandiu-se no mundo e a Pizza Hut realizou a primeira venda online em 1994, de uma pizza, obviamente (ABE, 2018). No Brasil, uma década e meia mais tarde, a primeira empresa a realizar vendas por meio de sites na internet foi a Livraria Cultura, cujas atividades nesse segmento iniciaram-se em 1995 (TOMÉ, 2018).

A participação de um maior número de indivíduos tem provocado algumas mudanças na forma de comercializar dentro do ambiente virtual. De início, as plataformas de varejo online eram exibidas como se fossem catálogos, onde o cliente poderia visitar as páginas e escolher o seu produto de interesse. Não havia muita interação entre consumidor e vendedor. Contudo, as empresas passaram a observar a crescente necessidade do consumidor em avaliar o produto após a aquisição, assim como fazer comentários sobre a sua experiência de compra dentro da própria plataforma de CE. A avaliação pós-compra oferece a oportunidade de a empresa conhecer a opinião do cliente, se ficou satisfeito com a experiência de compra ou se as suas necessidades não foram atendidas (CERIBELI; MERLO; CÂNDIDO, 2014).

Dentro desse ambiente de transformações, o CE com suas plataformas de varejo virtual ultrapassou problemas relacionados como a "confiança e informação por meio de feedback e sistemas de classificação e com a oferta de mecanismos de depósito em garantia e solução de controvérsias" (WORLD BANK, 2016, p.12). Dessa forma, o contato entre clientes, assim como a facilidade em disponibilizar um produto para venda, já tem provocado outros tipos de comércio eletrônico, como a chamada venda de consumidor para consumidor, rompendo o paradigma de que as transações comerciais ocorrem somente entre consumidores e empresas.

Possuir uma plataforma de CE tem deixado de ser um diferencial competitivo no mercado brasileiro, à medida que cresce o número da população inserida no ambiente virtual através do acesso à internet de banda larga. Outro fator que tem impulsionado as pessoas no uso da internet é a melhoria nos serviços de banda larga móvel no país que tem popularizado o acesso das camadas sociais de menor renda, 
as chamadas classe C e D (EBIT, 2016).

No Brasil, em especial, o segmento Business to Consumer - B2C tem se profissionalizado e crescido nos últimos 20 anos. Ressaltando que a adesão do CE está atrelada a maior disponibilidade da internet no país, no final da década dos anos noventa, o e-commerce começou a ganhar popularidade ao oferecer produtos como livros, CD e DVD. Las Casas e Pinheiro (2013, p. 44) apontam o surgimento de "um mercado amplamente segmentado com consumidores mais sofisticados e informados, cujas necessidades são cada vez mais específicas, reforçando a competição no varejo".

\subsection{A EVOLUÇÃO DO VAREJO VIRTUAL BRASILEIRO}

Com o passar do tempo, a diversidade de produtos e serviços ofertados no CE cresceu de forma que é possível comprar de pacotes turísticos a roupas e acessórios de moda. O CE brasileiro tem se consolidado e alcançado um maior número de consumidores. Um ponto a ser destacado é o tíquete médio de compra, que é o valor médio que cada cliente compra através da loja de varejo virtual (SEBRAE, 2016). O tíquete médio de compra no e-commerce brasileiro tem sido acompanhado pela empresa Ebit desde 2000.

No Gráfico1 apresenta-se um comparativo entre os dados coletados durante o primeiro semestre dos anos de 2011 a 2018. 


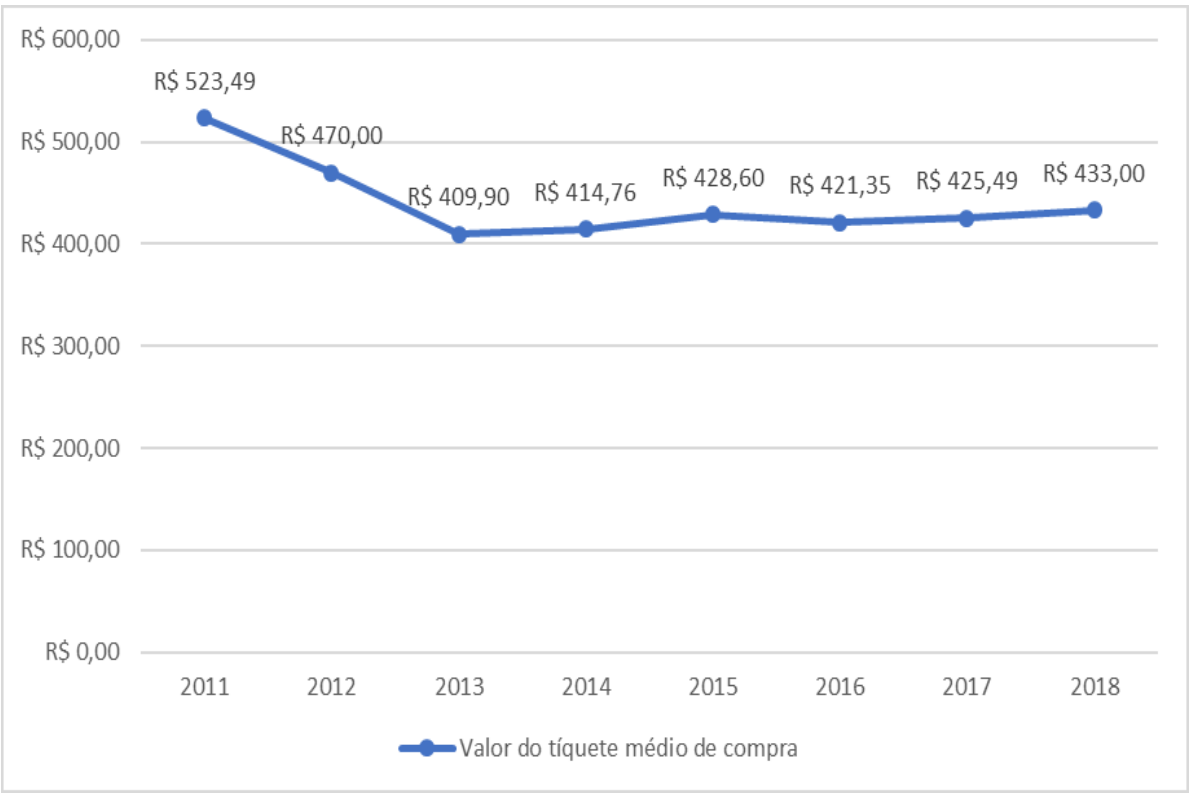

Gráfico 1. Evolução do tíquete médio de compras do CE no primeiro semestre dos anos de 2011 a 2018 (Reais)

Fonte: Adaptado da Ebit (2018).

Observa-se que houve diminuição no tíquete médio de compra entre 2011 e 2013, mas verifica-se uma retomada do crescimento no período seguinte mesmo diante da crise econômica que o país se encontra desde o ano de 2014 (IBRE, 2017). Embora o tíquete médio de 2018 se encontre abaixo dos valores registrados em 2011 e 2012, período anterior à crise econômica brasileira, observa-se lenta recuperação, mesmo nos anos de desempenho negativo da economia brasileira. A economia brasileira apresentou baixas taxas de crescimento ou negativas: $0,5 \%$ em 2014; (-) 3,5\% em 2015; (-)3,3 em 2016; 1,1\% em 2017; e 1,2\% em 2018 (IBGE, 2018). 


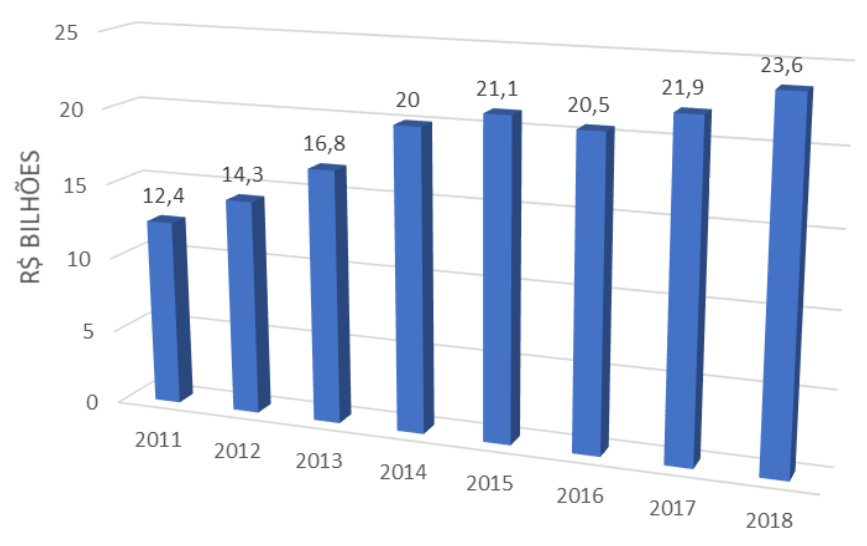

Gráfico 2. Faturamento do CE no primeiro semestre de 2011 a 2018 (milhões de reais) Fonte: Adaptado da Ebit (2018).

Verifica-se, por meio do Gráfico 2, que houve tendência de crescimento do faturamento no período, apesar do declínio ocorrido em 2016 em relação ao ano anterior, refletindo o comportamento do valor do tíquete médio e o desempenho econômico do país. Da mesma forma, existe uma tendência de crescimento nas vendas através do varejo virtual porque os usuários da internet e clientes estão realizando compras mais conscientes, assim como encontram a sua disposição informações que trazem uma mais economia de tempo e dinheiro com o uso de ferramentas que comparam preços - os chamados buscadores - e das redes sociais, que disponibilizam a opinião com a experiência de compras anteriores de diversos clientes (EBIT, 2017).

Conforme se verifica no Gráfico 3, o número de pedidos no CE apresentou crescimento de 3,9\% no primeiro semestre de 2017 quando comparado ao mesmo período de 2016. No primeiro semestre de 2016 houve uma retração de 1,8\% se comparado a 2015. 


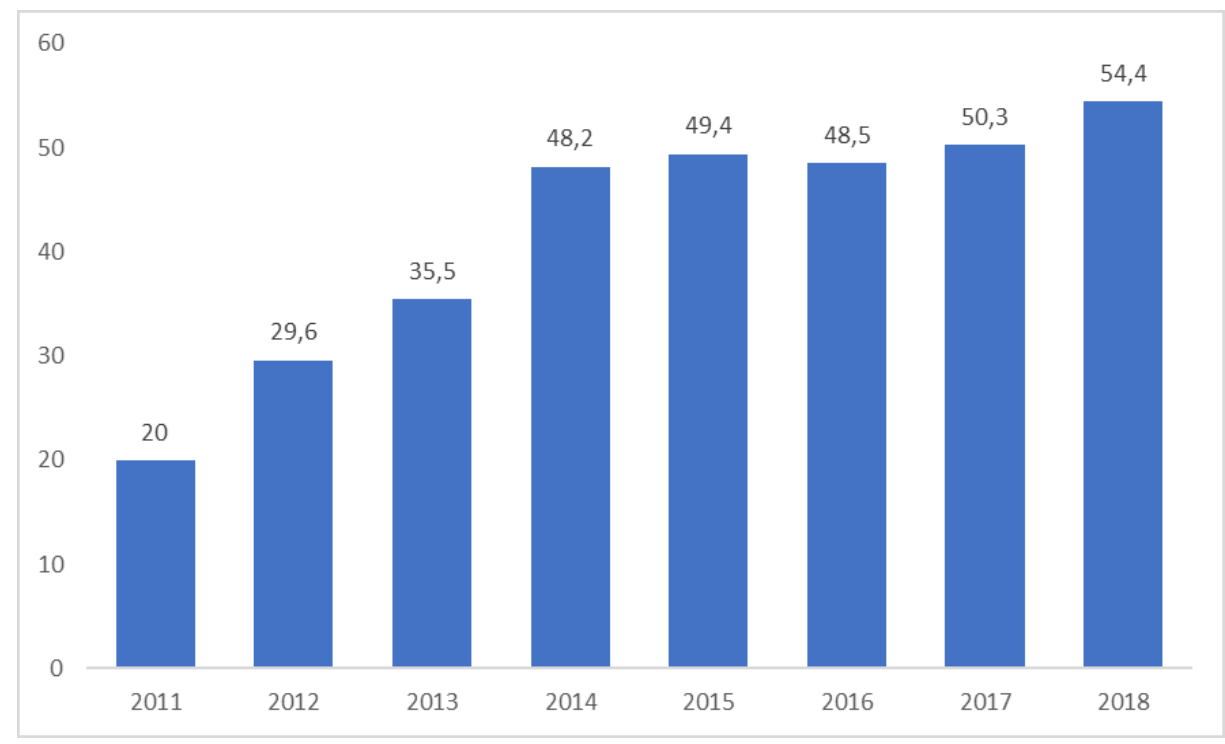

Gráfico 3. Número de pedidos no CE no primeiro semestre de 2011 a 2018 (milhões) Fonte: Adaptado de Ebit (2018).

Os dados apresentados permitem inferir que o faturamento e o número de pedidos indicam o mesmo comportamento, qual seja, de crescimento até 2015, redução em 2016 e retomada em 2017 e 2018. No entanto, o tíquete médio não retornou aos valores anteriores à crise econômica brasileira, revelando que um maior número de consumidores compra menores valores, indicando novo perfil de compradores, possivelmente.

\subsection{PERFIL DOS COMPRADORES DO VAREJO VIRTUAL BRASILEIRO}

Nos primeiros semestres dos anos de 2016, 2017 e 2018, a participação dos compradores no varejo virtual brasileiro é semelhante entre homens e mulheres, cabendo cerca de 50\% para cada em deles (EBIT, 2018). No que se refere aos dados referentes a faixa etária dos compradores, no Quadro 4 mostra-se que não houve alteração na idade média do e-cliente, de 43 anos. A maior parcela de compradores situa-se entre 35 e 49 anos. As duas últimas faixas etárias somam $69 \%$ do total, ou seja, três quartos dos compradores, aproximadamente, têm mais de 35 anos. 
Quanto a característica dos compradores no CE de acordo com a renda familiar no Brasil, observa-se no Quadro 5 a maior participação daqueles cuja renda familiar é de menos de $\mathrm{R} \$ 3.000$ /mês seguida pela faixa imediatamente superior, entre $R \$ 3.001$ e $R \$ 5.000 /$ mês. Somadas, essas duas faixas de renda perfazem o total de $61,3 \%$ e $57,1 \%$ do total de compradores em 2016 e 2017, no ano de 2018 perfaz o somatório de $46,4 \%$. A queda dessas faixas indica a maior participação dos estratos superiores de renda em 2018, destacando-se a faixa acima de $\mathrm{R} \$ 8.001$, cuja parcela passou de 20,8\% em 2016 para 24,5\% em 2017, já em 2018 houve uma queda para $17,8 \%$.

Quadro 3. Participação dos compradores no CE no ano de 2016, 2017 e 2018 de acordo com a renda mensal familiar brasileira

\begin{tabular}{|l|c|c|c|}
\hline Renda familiar/Ano & 2016 & 2017 & 2018 \\
\hline Menos de $\mathrm{R} \$ 3.000$ & $37,5 \%$ & $33,4 \%$ & $16,4 \%$ \\
\hline Entre $\mathrm{R} \$ 3.001$ e $\mathrm{R} \$ 5.000$ & $23,8 \%$ & $23,7 \%$ & $30 \%$ \\
\hline Entre $\mathrm{R} \$ 5.001$ e $\mathrm{R} \$ 8.000$ & $17,9 \%$ & $18,3 \%$ & $35,8 \%$ \\
\hline Acima de $\mathrm{R} \$ 8.001$ & $20,8 \%$ & $24,5 \%$ & $17,8 \%$ \\
\hline Renda familiar média & $\mathrm{R} \$ 5.409,00$ & $\mathrm{R} \$ 5.573,00$ & $\mathrm{R} \$ 6.475,00$ \\
\hline
\end{tabular}

Fonte: Adaptado de Ebit (2018).

Se comparada ao primeiro semestre de 2016, a renda média mensal familiar aumentou $7,7 \%$ potencializada pela diminuição do poder de compra da classe $C$ no comércio eletrônico, de forma que as classes A e B no primeiro semestre de 2017 aumentaram sua participação nas compras via e-commerce elevando a renda média familiar de $\mathrm{R} \$ 5.174,00 \mathrm{em} 2016$ para $\mathrm{R} \$ 5.573,00 \mathrm{em} 2017$ e no ano de 2018 para $\mathrm{R} \$$ 6.475,00 (EBIT, 2018).

Ressalta-se nesse quadro a queda da participação de compradores com renda familiar no estrato menos de $\mathrm{R} \$ 3$ mil, sobretudo no último ano analisado, passando a representar menos da metade da parcela dos anos anteriores. Associandose ao aumento do faturamento do CE brasileiro, assim como do tíquete médio e do número de pedidos que aumentaram nesses anos, entre 2016 e 2018, confirma 
que a crise econômica por que atravessa o país, "afeta mais os trabalhadores menos escolarizados e as famílias de menor renda” (IPEA, 2019, p.1).

No que se refere a participação dos compradores no CE brasileiro por macrorregião, no Gráfico 4 mostra-se que o Sudeste alcança maior presença, seguida pelo Sul. Entretanto, observa-se aumento da parcela relativa desta última região em detrimento das demais.



Gráfico 4. Participação dos compradores no CE de acordo com as regiões brasileiras - 2016 a 2018 Fonte: Adaptado de Ebit (2018).

Detendo mais de $60 \%$ do total de compradores do CE, na região Sudeste, representada pelos estados de São Paulo, Rio de Janeiro, Minas Gerais e Espírito Santo, concentram-se a maior parte das empresas de e-commerce. A sede das lojas de comércio eletrônico no Brasil distribui-se da seguinte forma nas diferentes macrorregiões: $58 \%$ no Sudeste; $18 \%$, no Sul; $12 \%$, no Nordeste; $3 \%$, no Nordeste (SEBRAE, 2017). Além disso, conta com o maior número de habitantes do país.

Ademais, tendo em vista de que o frete ainda possui um elevado custo no Brasil que, além das dimensões continentais que caracteriza o país, ainda enfrenta problemas de infraestrutura de transporte, o que acaba reduzindo o interesse de compra do consumidor de regiões mais afetadas, pois o frete pode superar o valor do produto adquirido em determinados casos (EBIT, 2018). 
Seguindo o mesmo comportamento apresentado nos anos anteriores, em 2018, saúde, cosméticos e perfumaria e moda e acessórios e respondem por cerca de $66 \%$ das categorias mais vendidas no CE brasileiros, em volume.

O mercado consumidor brasileiro de cosméticos, perfumaria e produtos de higiene pessoal é o quarto maior do mundo com participação de 6,9\% do mercado global, trás dos Estados Unidos, China e Japão (ABIHPEC, 2018). No setor do varejo em geral, o segmento da beleza destaca-se entre os dez principais. O público masculino tem aumentado o consumo destes produtos colocando o Brasil em segundo lugar neste segmento, no mundo (SEBRAE, 2019).

Quanto ao setor de moda e acessórios, é um segmento que atrai pequenos e microempreendedores, ocupando o sexto lugar em termos de faturamento no mercado brasileiro de CE (EBIT, 2017). A impossibilidade de se provar roupas e acessórias foi sendo suplantada pela disponibilização de imagens, tabela de medidas, descrições detalhadas e possibilidade de troca sem custos adicionais.

\section{CONCLUSÃO}

A disponibilização da internet banda larga facilitou a comunicação e os indivíduos passaram a se comunicar com maior frequência através por esse meio e ferramentas disponíveis nas redes virtuais. A viabilidade de acesso da população ao computador pessoal e aos dispositivos móveis como os smartphones e tablets dinamizaram a produção, troca de informações, compras e vendas. A ampliação e a melhor qualidade dos serviços ofertados foram fundamentais para o crescimento do CE no mundo e no Brasil.

Apesar das baixas taxas de crescimento e mesmo negativa da economia brasileira, o CE tem se mostrado positivo visto o desempenho do faturamento e do número de pedidos e mesmo do tíquete médio que vem se recuperando. O comprador brasileiro situa-se na faixa etária acima dos 35 anos e diferença de participação entre os sexos é mínima, sendo que a queda participação de compradores de estratos de renda inferiores é notória, nos últimos anos.

A região Sudeste do Brasil, além de abrigar o maior número de empresas 
de CE também concentra o maior número de compradores dessa modalidade de comércio. As categorias mais vendidas no CE brasileiro acompanham o padrão mundial, focalizados nas categorias saúde, cosméticos e perfumaria além de moda e acessórios.

\section{REFERÊNCIAS}

ABE, R. Inovação é potência motriz que move o e-commerce. 2018. Disponível em: https://www.ecommercebrasil.com.br/artigos/inovacao-e-potencia-motriz-quemove-o-e-commerce/. Acesso em: 21 jun. 2019.

ABIHPEC. Associação Brasileira de Higiene Pessoal, Perfumaria e Cosméticos. Anuário ABIHPEC 2018. São Paulo: ABIHPEC, 2018. Disponível em: https:// abihpec.org.br/anuario-2018-flip/mobile/index.html\#p=6. Acesso em: 21 jun. 2019.

ALBERTIN, A. L. Pesquisa FGV-EAESP de comércio eletrônico no mercado brasileiro. 18. ed. Fundação Getúlio Vargas: Rio de Janeiro, 2016.

ALBERTIN, A. L. Comércio eletrônico: modelo, aspectos e contribuições de sua aplicação. 6. ed. São Paulo: Atlas, 2010.

BERTAGLIA, P. R. Logística e gerenciamento da cadeia de abastecimento. 2. ed. São Paulo: Saraiva, 2009.

CATALANI, L. et al. E-commerce. 1. ed. Rio de Janeiro: FGV, 2008.

CERIBELI, H. B.; MERLO, E. M.; CÂNDIDO, A. Uma proposta de segmentação dos consumidores no comércio eletrônico. Nucleus, Ituverava, v. 11, n. 1, 2014.

COELHO, L. D. S.; OLIVEIRA, R. C.; ALMÉRI, T. M. O crescimento do e-commerce e os problemas que o acompanham: a identificação da oportunidade de melhoria em uma rede de comércio eletrônico na visão do cliente. Revista de Administração do Centro Universitário Salesiano de São Paulo- UNISAL, Campinas, v. 3, p. 63-85, Jan/Abr 2013. 
EBIT. Webshoppers. 34. ed. São Paulo, 2016.

EBIT. Webshoppers. 35. ed. São Paulo, 2017.

EBIT. Webshoppers. 36. ed. São Paulo, 2018.

FEE. Fundação de Economia e Estatística. Atualização de valores. 2017.

Disponivel em: http://www.fee.rs.gov.br/servicos/atualizacao-valores/. Acesso em: 20 fevereiro 2017.

IBGE. Instituto Brasileiro de Geografia e Estatística. PIB, 2018. Disponível em: https://www.ibge.gov.br/explica/pib.php. Acesso em: 21 jun. 2019.

IPEA. Instituto de pesquisa econômica aplicada. Desemprego de longo prazo cresce 42,4\% entre 2015 e 2019. 2019. Disponível em: http://agenciabrasil.ebc. com.br/economia/noticia/2019-06/desemprego-de-longo-prazo-cresce-424-entre2015-e-2019. Acesso em: 21 jun.2019.

KALAKOTA, R.; WHINSTON, A. Eletronic commerce: a manager's guide. New York: Addison-Wesley, 1997.

KOTLER, P.; KARTAJAYA, H.; SETIAWAN, I. Marketing 3.0: as forças que estão definindo o novo marketing centrado no ser humano. Rio de Janeiro: Elsevier, 2010.

LAS CASAS, A. L.; PINHEIRO, W. Correlação entre produção científica e pesquisa de mercado sobre o comércio eletrônico no Brasil. Universitas Gestão e TI, Braślia, v. 3, n. 2, p. 43-51, ju-dez 2013.

NASCIMENTO, R. M. D. E-commerce no Brasil: perfil do mercado do e-consumidor brasileiro. 1. ed. Rio de Janeiro: FGV, 2011.

SCANDIUZZI, F.; OLIVEIRA, M. M. B.; ARAÚJO, G. J. F. D. A logística no Comércio eletrônico B2C: um estudo nacional multicasos. Revista de Estudos do CEPE, Santa Cruz do Sul, v. 34, p. 231-241, Jul-Dez 2011.

SEBRAE. Serviço Brasileiro de Apoio às Micro e Pequenas Empresas. $3^{\text {a }}$ pesquisa 
nacional de varejo online. SEBRAE e E-commerce Brasil: Brasília, 2016.

SEBRAE. Serviço Brasileiro de Apoio às Micro e Pequenas Empresas. Mercado de cosméticos. 2019. Disponível em: http://www.sebraemercados.com.br/mercadode-cosmeticos/. Acesso em: 21 jun. 2019.

TOMÉ, L. M. Comércio eletrônico. Caderno Seytoriaç. Banco do Nordeste. Ano 3, n. 43, set. 2018. Disponível em: https://www.bnb.gov.br/ documents/80223/4049480/43_Ecommerce_2018.pdf/2ad80a68-847f-cda8-34b44f3f27496339. Acesso em: 21 jun. 2019.

VENETIANER, T. Como vender seu peixe na internet: um guia prático de marketing e comércio eletrônico. 3. ed. Rio de Janeiro: Campus, 2000.

WORLD BANK. 2016 International Bank for Reconstruction and Development. Washington DC, 2016.

Recebido em: 25/03/2019 Aceito em: 04/07/2019 